year and than each 6 month. The main outcome measures in RA were DAS 28, $\mathrm{HAQ}$, EuroQuol, SF36, CRP, in AS BASDAI, HAQ and ASDAS. Reasons for drug discontinuation were reported as primary failure, secondary failure, adverse advent, remission and others. Statistics - survival on therapy was estimated by Kaplan-Meier analysis. The search for outcome predictors was performed by logrank test (continuous predictors were appropriately categorized).

Results: Altogether 3159 patients with RA, 1785 with AS and 723 with PsA were included.

1598 patients with RA were treated with adalimumab and retention was $75,8 \%$ in one year, $43,8 \%$ in 5 years and $27,7 \%$ in 10 years. The reasons for drug discontinuation were: primary failure $24,9 \%$, secondary failure $30,5 \%$, and adverse events $(19,8 \%)$

Predictive factors for adalimumab retention in rheumatoid arthritis were: younger age $<50$ years, failure of $\leq 1$ csDMARDs in past, combination therapy with csDMARDs at baseline. Sex, RF and anti CCP were not predictive. Drug retention was longest in AS (median 99,6 m), than PsA (median 62,5 m) and shortest in RA (median 43,9 m) (graph 1). Drug survival in rheumatoid arthritis was longer on etanercept than on infliximab $(p<0,001)$, longer on adalimumab than infliximab $(p<$ $0,001)$ and equal between adalimumab and etanercept $(p=0,85)$.

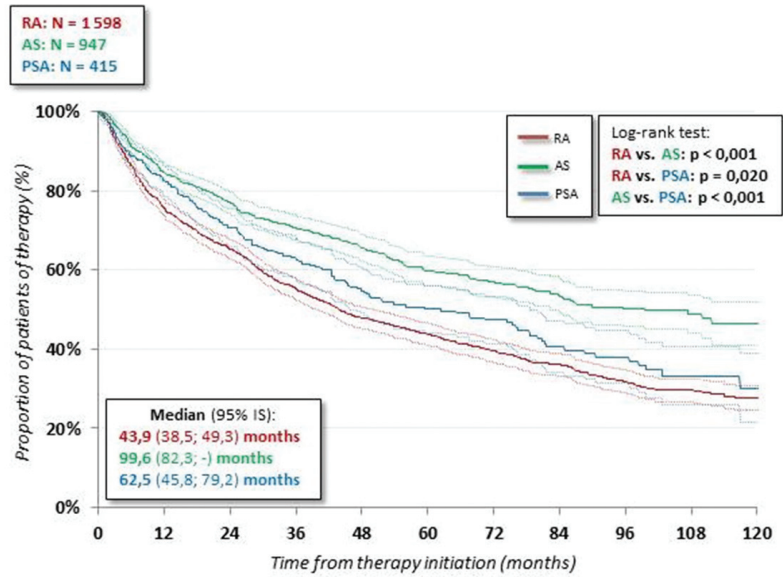

Conclusions: Adalimumab retention in registry ATTRA was comparable to other European registries. Predictors of drug retention in RA were: younger age $(<50$ years), $\leq 1$ csDMARDs in past and in combination with csDMARDs. Survival on adalimumab was longer in AS, than PsA and RA and the same was true for all three anti TNF drugs. Survival on drug in rheumatoid arthritis was longer in adalimumab and etanercept compared to infliximab.

References: Key words: biologic therapy, registry ATTRA

Disclosure of Interest: None declared

DOI: 10.1136/annrheumdis-2018-eular.2407

\section{FRI0123 COMPARATIVE EFFECTIVENESS OF SUBCUTANEOUS VERSUS INTRAVENOUS TOCILIZUMAB IN A PAN- EUROPEAN COLLABORATION OF REGISTRIES}

K. Lauper ${ }^{1,2}$, M.J. Santos ${ }^{3}$, on behalf of Rheuma.pt, F. lanonne ${ }^{4}$, Z. Rotar ${ }^{5}$, D. C. Nordström ${ }^{6}$, C. Codreanu' ${ }^{7}$, T.K. Kvien ${ }^{8}$, E.K. Kristianslund ${ }^{8}$, K. Pavelka ${ }^{9}$, G. Lukina ${ }^{10}$, M.V. Hernández ${ }^{11}$, S.L. Gale ${ }^{12}$, K. Sarsour ${ }^{12}$, Y. Luder ${ }^{13}$, D. S. Courvoisier ${ }^{1}$, C. Gabay ${ }^{1,2}$. ${ }^{1}$ Rheumatology, University Hospitals of Geneva, Geneva; ${ }^{2}$ SCQM, Zürich, Switzerland; ${ }^{3}$ Rheumatology Department, Hospital Garcia de Orta, Almada, Portugal; ${ }^{4}$ GISEA, University Hospital of Bari, Bari, Italy; ${ }^{5}$ BioRx.si, University Medical Centre Ljubljana, Ljubljana, Slovenia; ${ }^{6}$ ROB-FIN Helsinki University Hospital and Helsinki University, Helsinki, Finland; ${ }^{7}$ Center of Rheumatic Diseases, University of Medicine and Pharmacy, Bucharest, Romania; ${ }^{8}$ Diakonhjemmet Hospital, Oslo, Norway; ${ }^{9}$ Charles University, Prague, Czech Republic; ${ }^{10}$ ARBITER, Institute of Rheumatology, Moscow, Russian Federation; ${ }^{11}$ Rheumatology, Hospital Clinic Barcelona, Barcelona, Spain; ${ }^{12}$ Genentech, South San Francisco, CA, USA; ${ }^{13} \mathrm{~F}$. Hoffmann-La Roche, Basel, Switzerland

Background: In randomised controlled trials, subcutaneous tocilizumab (TCZ sc) has been found non-inferior to intravenous TCZ (TCZ iv) for the treatment of rheumatoid arthritis (RA) patients. However, to our knowledge, there are no observational studies comparing these two different routes of administration in routine care.

Objectives: To compare the real-world effectiveness of TCZ Sc and TCZ iv in RA patients.

Methods: We included RA patients treated with TCZ from 8 European registries. We compared drug retention using Kaplan-Meier and Cox models. The proportions of patients achieving CDAl remission and low disease activity (LDA) at
1 year were compared using LUNDEX correction with computation of confidence intervals by bootstrapping. ${ }^{1}$

Results: 2896 patients were retrieved from the collaborative registries before January 2017, including 2469 TCZ iv and 427 TCZ sc. Baseline demographics were similar between both groups, but patients in the TCZ iv group had a more severe disease activity, with higher DAS28, CDAI, tender joint count (TJC), swollen joint count (SJC), ESR and physician global assessment values (table 1). Crude median retention was 2.14 years $(95 \% \mathrm{Cl} 2.03-2.33)$ for TCZ iv and 1.00 year for TCZ sc $(95 \% \mathrm{Cl} 0.83-1.10), \mathrm{p}<0.001$. However, in a covariateadjusted analysis, stratified by country- and year of treatment initiation, we found that hazards of discontinuation were similar among patients on TCZ iv compared to patients on TCZ sc (hazard ratio: 0.92 , CI 95\% 0.77-1.09). The average adjusted CDAl change at 1 year was -1.15 for TCZ iv, and -1.06 for TCZ SC patients ( $p$-value of interaction between treatment group and time: 0.68 ). The average adjusted DAS28 change at 1 year was also similar between groups ( -0.28 for TCZ iv and -0.09 for TCZ sc, $p$ value of interaction: 0.21$)$. CDAI remission and LDA at 1 year (LUNDEX corrected) were similar between TCZ sc and TCZ iv patients (CDAl remission: $9.5 \%$ in TCZ iv vs. $9.4 \%$ in TCZ sc (difference: $-0.1 \%$, bootstrap $95 \% \mathrm{Cl}:-3.8 \%>3.8 \%$ ); CDAI LDA: $37.3 \%$ in TCZ iv vs. $33.7 \%$ in TCZ sc (difference: $-3.6 \%$; bootstrap 95\% Cl: $-9.4 \% ; 2.5 \%$ )). Likewise, DAS28 remission and LDA at 1 year (LUNDEX corrected) were comparable between TCZ SC and TCZ iv (difference in DAS28 remission: $-2.7 \%$; bootstrap 95\% Cl: $-8.8 \%$; 3.5\%; difference in DAS28 LDA: $-5.8 \%$; bootstrap $95 \% \mathrm{Cl}$ : $-11.9 \% ; 0.4 \%)$

Abstract FRI0123 - Table 1. Baseline characteristics

\begin{tabular}{|c|c|c|c|}
\hline$N=2896$ & TCZ i.v $(n=2469)$ & TCZ s.c. $(n=427)$ & p \\
\hline Number of visits (median [IQR]) & $2[1-5]$ & $2[1-3]$ & \\
\hline Total patient-years & 5099.16 & 379.11 & \\
\hline Age, yr (median [IQR]) & $56.2[48.0,64.0]$ & $55.6[47.1,63.6]$ & 0.30 \\
\hline Female gender, $\mathrm{n}(\%)$ & $2004(81.2)$ & $345(80.8)$ & 0.91 \\
\hline Ever smoker, $\mathrm{n}$ (\%) & $319(21.7)$ & $68(22.1)$ & 0.94 \\
\hline BMI (median [ IQR]) & $25.3[22.4,28.6]$ & $25.2[22.7,28.5]$ & 0.73 \\
\hline Disease duration, yr (median [IQR]) & $7.8[3.4,14.7]$ & $8.0[3.4,15.7]$ & 0.52 \\
\hline Seropositivity (RF and/or ACPA), $n$ (\%)) & $1799(83.1)$ & $291(81.5)$ & 0.52 \\
\hline Previous bDMARDs, $n(\%)$ & $1750(75.0)$ & $302(73.5)$ & 0.54 \\
\hline Previous csDMARDs, $\mathrm{n}(\%)$ & $1939(89.4)$ & $345(90.1)$ & 0.76 \\
\hline Glucocorticoids, $n$ (\%) & $1276(51.7)$ & $237(55.5)$ & 0.16 \\
\hline Glucocorticoids dose, mg/day (median, IQR) & $5.0[5.0,10.0]$ & $5.0[5.0,8.0]$ & 0.17 \\
\hline Concomitant csDMARD & & & 0.40 \\
\hline None & $680(27.6)$ & $131(30.7)$ & \\
\hline MTX & $977(39.6)$ & $152(35.6)$ & \\
\hline MTX + other csDMARDs & $348(14.1)$ & $60(14.1)$ & \\
\hline Other csDMARDs than MTX & $462(18.7)$ & $84(19.7)$ & \\
\hline DAS28 (median [IQR]) & $4.6[3.2,5.6]$ & $4.2[2.6,5.1]$ & $<0.001$ \\
\hline CDAI (median, [IQR]) & $25.0[14.0,37.3]$ & $20.6[10.3,31.6]$ & $<0.001$ \\
\hline HAQ (median, [IQR]) & $1.4[0.9,1.9]$ & $1.3[0.8,1.9]$ & 0.17 \\
\hline TJC (over 28 joints) (median, [IQR]) & $8.0[3.0,14.0]$ & $5.0[2.0,11.0]$ & $<0.001$ \\
\hline SJC (over 28 joints) (median, [IQR]) & $6.0[2.0,11.0]$ & $4.0[0.0,8.0]$ & $<0.001$ \\
\hline ESR (mm/hour) (median, [IQR]) & $26.0[9.0,46.8]$ & $20.0[8.0,40.0]$ & 0.049 \\
\hline CRP (mg/L) (median, [IQR]) & $6.0[1.1,22.0]$ & $5.0[1.0,19.0]$ & 0.11 \\
\hline Patient global assessment (median, [IQR]) & $6.0[4.0,8.0]$ & $6.0[3.9,7.5]$ & 0.40 \\
\hline Physician global assessment (median, [IQR]) & $5.0[3.0,7.0]$ & $4.4[2.0,6.3]$ & 0.002 \\
\hline
\end{tabular}

Conclusions: Drug retention and clinical effectiveness, assessed by CDAI and DAS28 changes and responses, were similar in both groups of patients, treated with TCZ sc or TCZ iv.

\section{REFERENCE:}

[1] Kristensen LE, et al. Arthritis Rheum 2006;54:600-6. doi:10.1002/ art.21570

Disclosure of Interest: K. Lauper: None declared, M. Santos: None declared, F. lanonne: None declared, Z. Rotar: None declared, D. Nordström Grant/research support from: AbbVie, BMS, MSD, Pfizer, Roche and UCB, Consultant for: AbbVie, BMS, MSD, Roche, UCB and Pfizer, C. Codreanu: None declared, T. Kvien: None declared, E. Kristianslund: None declared, K. Pavelka Grant/research support from: AbbVie, Roche, Medis, MSD and Pfizer, Consultant for: AbbVie, Roche Amgen, MSD, BMS, UCB and Egis, G. Lukina Consultant for: BMS, Roche, MSD, AbbVie and Pfizer, M. Hernández: None declared, S. Gale Employee of: Genentech, K. Sarsour Employee of: Genentech, Y. Luder Employee of: F. Hoffmann-La Roche, D. Courvoisier: None declared, C. Gabay Grant/research support from: Roche, AbbVie, MSD and Pfizer, Consultant for: AbbVie, BMS, Roche, Pfizer, Celgene, MSD, Janssen Cilag, Amgen, UCB DOI: 10.1136/annrheumdis-2018-eular.2886 